\title{
Haptic Perception with a Robot Hand: Requirements and Realization
}

\author{
Paul Michelman \\ Department of Computer Science \\ Columbia University
}

\author{
Peter K. Allen \\ Department of Computer Science \\ Columbia University
}

\begin{abstract}
This paper first discusses briefly some of the recent ideas of perceptual psychology on the human haptic system particularly those of J.J. Gibson and Klatzky and Lederman. Following this introduction, we present some of the requirements of robotic haptic sensing and the results of experiments using a Utah/MIT dexterous robot hand to derive geometric object information using active sensing.
\end{abstract}

\section{Introduction}

During the last decade, a number of dexterous robot hands have been designed and manufactured [JIK+86] [UPB88] [Sal82]. Two tasks for which dexterous hands will ultimately prove superior to other types of robotic end effectors are related to general-purpose grasping and sensing complex objects. First, like human hands, they are general-purpose manipulators and therefore can be used for a variety of tasks because of their great number of degrees of freedom. Second, robotic hands can be used as complex sensors capable of detecting detailed information about object features not obtainable by other means. The future will see the use of robotic hands that can adapt themselves to a variety of different tasks and use tools with human-like dexterity, as well as employ a suite of sensors to help the hand gain detailed knowledge about the world with which it interacts.

Robotic hands will be required to in teract with unknown objects in complex environments. They will need sophisticated control and perceptual capabilities to enable them to gain object information from local cues. Prior to grasping an object, a robot hand will be called upon to make inferences concerning the object's precise shape and location. This information will be used to deduce possible grasping points. People use their hands to gather information about the shape, size, weight, texture, and material composition of objects. Although there is not a single sensor dedicated to the so-called sense of touch, or haptic sense, we use a wide variety of sensors and strategies to make inferences about the world. 
The use of active touch in robotics is still in it infancy. Only recently have multifingered, dexterous hands capable of fine manipulation been developed, and there is no consensus yet on the type of sensors needed for a robotic haptic system. Some of these sensors are beginning to be used, such as tactile sensing and force sensing. On another level, the interpretation of sensor data, no matter how fine, is a difficult task.

This paper discusses briefly some of the recent ideas of perceptual psychology on the human haptic system particularly those of J.J. Gibson [Gib66] and Klatzky and Lederman [KL88]. Following this introduction, we present some of the requirements of robotic haptic sensing and the results of experiments using a Utah/MIT dexterous robot hand to derive geometric object information using active sensing.

\section{The human haptic system}

J.J. Gibson was one of the first to discuss the active nature of touch. He realized that the world is not perceived passively, but with the active participation of a subject that uses its hands for "groping, prodding, palpating, hefting, rubbing and fingering." People use touch to sense the world and to change it in the process. Among other things, touch is used to infer the shapes of objects and their surface textures. In his experiments, subjects were asked to identify various shapes using touch alone, without the aid of vision. Symmetric objects proved too easy to most subjects, so a set of oddly shaped, unfamiliar objects was produced. Gibson noted that most subjects tried to identify the objects by performing a similar set of procedures. The three most typical were: (1) curving the fingers around the object and trying to fit fingers into cavities; (2) moving the fingers over the surfaces in some exploratory way; (3) tracing the curvature using opposition of thumb and finger or using a single finger. The discrimination of object shape is a function of the combination of sensing both the angles that the bones make with one another and the location of skin contacts.

More recent work on active touch has been done by Klatzky and Lederman [KL88], who formalized the findings of Gibson and attempted to taxonomize the procedures that people use during active touch. People use purposive movements during object exploration, and the haptic system is extremely good at 3-D object recognition. In experiments in which people were asked to identify 100 familiar manipulable objects without the aid of vision, they were able to recognize them at a rate of $96 \%$ with a latency time of $1-2$ seconds. The method that the subjects used was to probe each object testing fixed properties with so-called "exploratory procedures (EP)." Each EP is used to extract a different type of feature from an object. The principle EP's are: lateral motion to extract texture; pressure to study hardness; static contact for thermal sensing; unsupported holding for weight; enclosing for volume; and contour following to extract precise contour information as well as global shape. 


\section{Robotic haptic systems}

The fundamental building blocks of a robotic system for haptic perception are a dexterous hand with many degrees of freedom, different types of cutaneous and kinesthetic sensors, and a controller to reason about sensory input and plan movements. As previously stated, there are currently several devices that have many degrees of freedom and can be used for research in active sensing with a multifingered hand. Problems related to sensing, control and reasoning are still paramount, however.

\subsection{Sensing requirements}

In order to build hands for dexterous manipulation, progress must be made with respect to the hand's capability to perceive object features, such as weight, texture, hardness, and shape. If we were to model the haptic systems of robotic hands on the human system, we would see that the system would need to have the following classes of sensors: (a) tactile sensors that form a skin-like surface, (b) joint angle sensors to judge the relative positions of the different links (bones), and (c) muscle or tendon force sensors. Sensing joint angles and tendon tensions is possible with current technology, and several dexterous hands have incorporated these sensors successfully [JIK +86$]$ [Sal82]. The design and use of tactile sensing has proved more difficult, however.

The ideal tactile sensing system for a haptic system - one that mimics the human system - would be capable of detecting position, orientation, velocity, and forces [GHM89] [Har82] [Igg82]. The various types of receptors in human skin are each sensitive to different stimuli. Two main types are rapidly adapting (RA) mechanoreceptors, which are excited only by movement on the skin's surface and whose output encodes the velocity of movement: and slowly adapting (SA) mechanoreceptors, which respond to displacement of the skin, but, unlike the RA receptors, sustain a discharge even when the skin is held in a fixed position. Among the SA receptors are the Merkel cells, which respond to both compression and shear stimuli.

Artificial haptic perception would need to make use of similar types of receptor input. Spatial resolution of approximately $2 \mathrm{~mm}$ and pressure thresholds of less than $1 \mathrm{~g}$ have been reported for the tips of the human index finger. The sensors would be able to sense both normal and shear forces. They would also be compliant to allow them to register indentations as well. To be able to sense velocities as well as human skin (up to $1000 \mathrm{~Hz}$ ), the sensors would need high bandwidth. In addition to these operational characteristics, the artificial skin would need mechanical properties that would make it useful for robotics. Three important such mechanical properties are elasticity and conformability to the shape of the hand and an outer covering with high friction to help maintain stable grasps. Ultimately, we would like to see the development of a skin that covers the robot and provides this wide range of sensory inputs.

Many types of tactile sensor have been designed in recent years. but none has all of the properties required for a rich haptic system. Most of the sensors are capable of yielding only information about the locality and magnitude of contacts normal to point of contact, even 
though their sensitivity to forces and spatial resolution are good [Spe88] [Tis88] [Hil82]. Howe [HC89] has developed a sensor that can detect acceleration - which is important if a hand is to sense slip and texture. But no single sensor or sensor system can extract all of the features that a human haptic system can. Furthermore, one of the most important features required of a tactile sensing system is that it provide complete coverage of the area being sensed and that it conform to hand's shape. Bicchi, Salisbury, and Dario [BSD89] have experimented with an idea they call "intrinsic tactile sensing," in which force sensors located in the finger joints can be used to calculate the direction and magnitude of an applied force. Likewise, thermally sensitive skins have yet to be developed. Most tactile sensors are in the development stage, however, and have not yet been tested in a working haptic system.

Looking at the EP's of Klatzky and Lederman, we can see that each procedure makes use of different types of mechanoreceptor. The EP's and corresponding mechanoreceptor type are summarized in Table 1. It is clear that given current technology and the availability of sensors to detect normal forces, it is possible to develop EP's to detect at least hardness, weight, volume and contour information.

\begin{tabular}{||l|l|l||}
\hline EP & Quality & Mechanoreceptor \\
\hline \hline Lateral motion & Texture & Accelerator type \\
\hline Pressure & Hardness & Force sensors \\
\hline Static contact & Thermal sensing & Thermal sensors \\
\hline Unsupported holding & Weight & Force sensors \\
\hline Enclosing & Volume & Joint angles \& tactile force \\
\hline Contour following & Contour information & Joint angles \& tactile force \\
\hline
\end{tabular}

A haptic system requires a convergence of many sensory inputs to form inferences about object properties. When a hand makes contact with an object, there are many cues that an intelligent planner can use and must be looking for. For example, suppose that a single finger is moving toward a hard surface, but the height of the surface is unknown. When the finger touches the surface, several changes in the system state occur. If the finger has tactile sensors at the point of contact, these sensors will provide the exact location of the contact region. Even without tactile contact, it is possible to realize that the finger is touching the surface by means of other sensors. By interrogating the joint angle sensors on the finger, the controller detects that further movement commands produce no effect. Therefore, an obstacle must be hindering motion and contact can be hypothesized. Similarly, if the finger's motion is controlled by flexor and extensor tendons, and the forces on these tendons are monitored, a sudden change in the values of these tendon forces is a certain indication of contact. Another technique would be to use the intrinsic tactile sensors mentioned above. The human haptic system uses all of these cues, as well as expectations about the environment, to formulate hypotheses about object features, and it will be important for dexterous robot hands to fuse the available sensory inputs. In addition, the fusion of many types of sensory input makes the sensing more robust and reliable.

Stansfield [Sta86] experimented with feature extraction using a single tactile sensor mounted on a robot arm. The sensor used was an LTS 200 from LORD Corporation. Although this sensor allowed the straightforward detection of surface normals with a single reading, it is not suitable for use on dexterous hand, which often requires multiple sensing points to infer a 
surface normal. Following Klatzky and Lederman, the system successfully determined several interesting object properties. (1) Compliance: $A$ force $F$ was applied to an object and the position of the sensor noted. Then the sensor was moved toward the object surface until a force $2^{*} F$ on the sensor was developed and the second position was noted. The larger the distance between the two positions, the greater the compliance of the object. This technique appeared to work reliably for a number of materials, such as wood, a sponge, and a folded cloth. (2) Elasticity: Elasticity is a measure of how well a material returns to its original position after being pressed. In this procedure, a material is compressed, with original and final positions noted. Then the sensor is removed from the material completely. Finally, the sensor is returned to the location of the initial contact and a third position is noted. If the third reading is the same as the first, the material is considered elastic. (3) Texture: An attempt was made to detect different textures using a single tactile impression. If the sensor is placed parallel to an object's surface and a large portion of the sensor array detects contact, then the surface is considered smooth. If only a few contacts are noted over a wide range, then the surface is thought to have a rough texture. This method is not robust, however, and a more active approach to texture, probably one using a different type of sensor, is required. Stansfield also used her system to detect what she called "derived features," such as edges, corners, and contours.

\subsection{Control requirements}

Given a set of sensors, how can we reason about their inputs? The system controller for tasks of object recognition will be composed of at least (1) a high-level reasoning and planning module; (2) a module that converts the high-level plans into lower-level movement primitives and feeds the commands to the robot; (3) a knowledge database that the high-level reasoning module has access to.

- The high-level reasoning and planning stage generates hypotheses about an object's shape and produces a sequence of steps to help refine these hypotheses. This module has access to all sensor data and maintains information about the current environment. In addition, it makes use of a knowledge base that contains information about known objects. This module decides which exploratory procedures to use.

- The middle-level module converts the gross motion plans of the high-level module to fine motion commands and sends them to the robot controller. The outputs from this module are movement primitives (joint trajectories, for example) and the activation of sensing processes to monitor the environment.

- A knowledge base is required if object recognition is to be performed [TBK87]. The knowledge database stores information about objects' geometric properties as well as material features. The knowledge base should use multiple representations for the same object. Certain types of reasoning and planning are more natural with one type of representation than another; so it is often desirable to store different representations of a single object in the database. For example, a single object may be represented in an analytic, volumetric form. such as superquadrics (see below), and also be stored as a set 
of polygonal faces. The volumetric representation may be suitable for the early stages of shape recovery, while the detailed polygonal representation may be more applicable in searching for grasping points.

As an example of a complete system, suppose our system is given the task of recognizing the shape of a rectangular block. Furthermore, suppose an accompanying vision system has given a rough idea of its size, position and orientation. The reasoning and planning module plans a strategy to decide what the object is. It first generates a hypothesis and then selects a sequence of exploratory procedures to test the hypothesis. The middle-level controller converts the high-level plan into a sequence of commands to move the robot and activate the required sensors. In this example, the planner might use the enclosing exploratory procedure if, after analyzing the vision input, it concludes that the extent of the object is not much larger than the hand. This exploratory procedure is understood by the middle-level controller to be composed of a sequence of movements, such as "open the hand to a desired position," then "close the hand until all fingertips make contact with the object." These motions are translated into actual joint angles, and at the same time, sensing processes must be activated so that the moment of contact can be noted. Once the enclosing EP is complete, the reasoning module has access to a set of contact points which it can use to further refine its hypothesis about the type of object under observation, and another procedure, such as surface following, can be selected.

\section{Active sensing with a Utah/MIT hand}

At Columbia University, we have begun the development of a system that can infer the shapes of objects using active touch sensing with a multifingered hand. Although nothing in our work precludes the use of vision, to date we have worked solely with blind touch to gain geometric object information.

\subsection{System Overview}

We have built a system that consists of a Utah/MIT hand attached to a PUMA 560 manipulator [AMR89]. The hand has four fingers, each with four degrees of freedom. The position of each joint is controlled by two tendons, extensor and flexor. The hand resembles the human hand in size and shape, but lacks a number of features that humans find very useful. In particular, it has no palmar degree of freedom (closing of the palm) and the thumb is placed directly opposite the other three fingers, with all fingers identical in size (see Figure 1). The hand has joint position sensors that yield joint angle data and tendon force sensors that measure forces on each of the $t$ wo tendons that control a joint. The PUMA adds 6 degrees of freedom, yielding a 22 degree-of-freedom system. To control the system in real time, we use the embedded controllers in each of these systems, controlling and communicating with them through an intelligent highlevel controller that links together the movements of the arm, hand, and fingers with feedback sensing of joint positions, tendon forces, and tactile responses on the fingers. Tactile sensors (described below) are mounted on the hand's fingertips. 


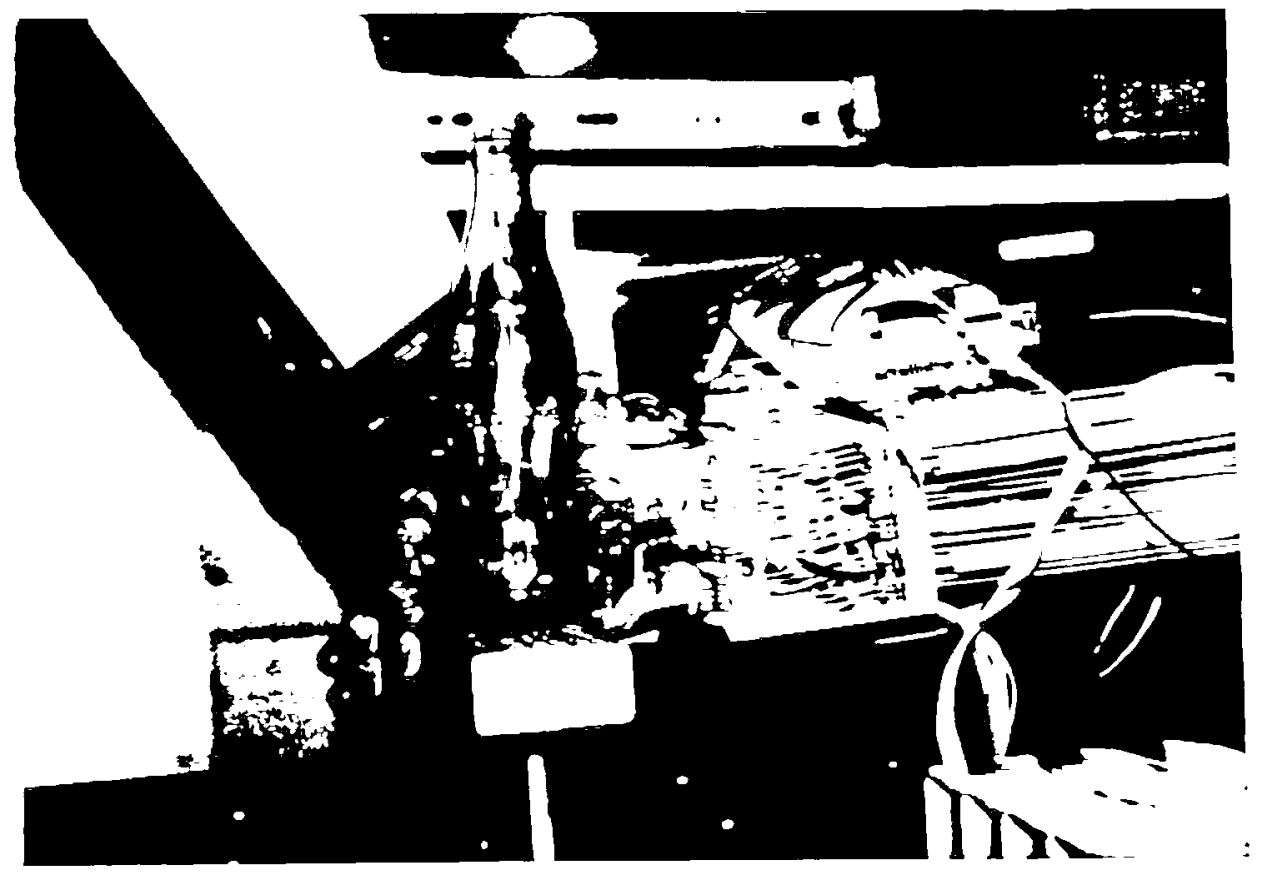

Figure 1: Utah/MIT hand with tactile sensors mounted

The hardware structure of the system is shown in Figure 2. The high-level control resides in a SUN-3 processor. The SUN serves as the central controller, and has access to a UNIX-based system for program development and debugging. Kinematic and inverse kinematic analysis is handled by a dedicated 68020 board. An analog controller is responsible for the low-level servo control of the system. The tactile sensing system is controlled by another dedicated 68020 that monitors the forces on each of the sensor pads.

We have mounted tactile sensors on each of the hand's fingers. The pads we are using [Co.86] are constructed by sandwiching a piezoresistive polymeric material between two pliable sheets of Kapton. The application of forces on the pads provides an increased electrical flow channel between the two sheets as the material is compressed. The piezoresistive polymer is patterned to form rows on one substrate and columns on the other. The rows and columns form a grid in which each intersection acts as a force-sensitive variable resistance whose value decreases approximately exponentially with normal force. The pads consist of 16 rows by 16 columns, providing a sense resolution of 256 points on a $0.5 \times 1.0$ inch $(0.63 \mathrm{~cm} \times 1.25 \mathrm{~cm})$ pad. The pads have excellent signal isolation. The 256 sites of each sensor pad are addressed independently by analog circuitry that cancels current flow in all paths of the grid except the one containing the resistive element being measured using a method developed by van Brussel and Belien [vBB86].

Currently, the sensors are mounted on the hand's fingertips, and they are connected by a cable to the circuitry that handles signal multiplexing and $A / D$ conversion. We scan all 


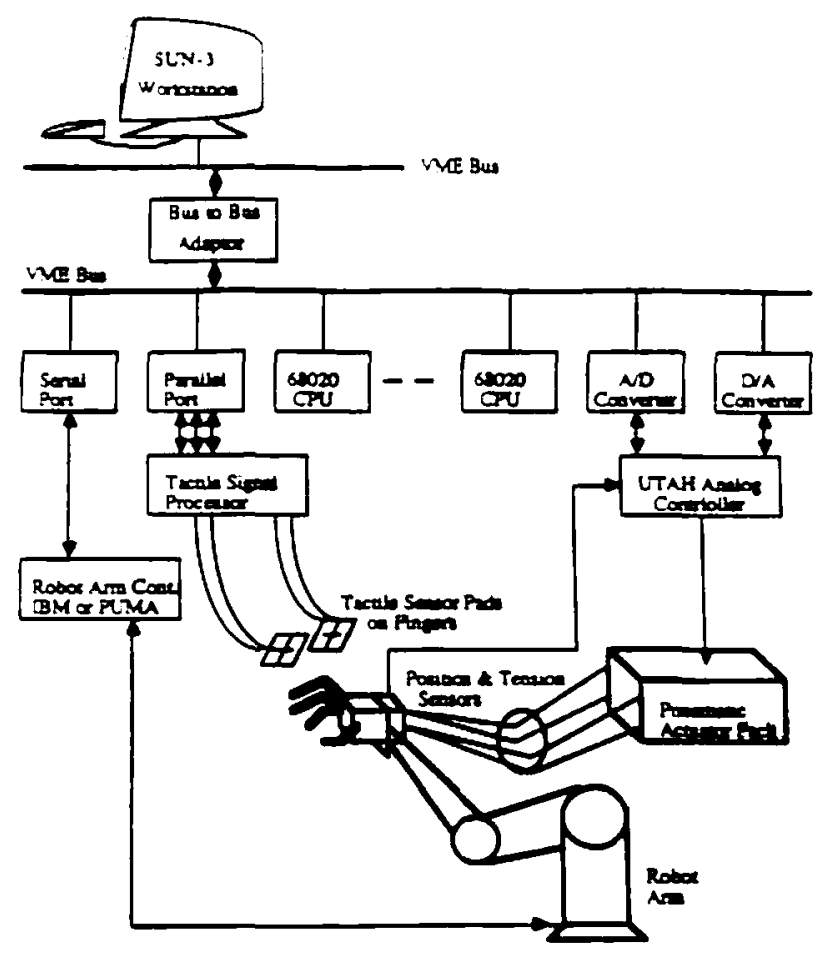

Figure 2: Hardware structure of system

256 elements of each sensor and analyze the tactile image to detect whether or not there is contact. If there is contact, then the first moments of the image are taken to determine the size of the contact area and its center. We have been able to get errors of less than $2 \mathrm{~mm}$ using this technique. There are several difficulties with this type of tactile sensor, however. Although the Kapton which forms the sensor is flexible, the pads do not conform to shape of the fingertip, which is not a developable surface. Sensors embedded in more elastic medium would be allow for better conformability. In part due to their lack of conformability, but also due to their size, the Interlink sensors cover only a small portion of each finger. They do not cover the outermost portion of the fingertip, and they do not cover the surfaces around the joints.

A three layered programming environment has been developed to control the hand and arm [AMR89]. On the first level, we have developed primitives to control rudimentary hand and arm movements and to gather sensor data from all of the sensors (joint angle, tendon tension, arm position, and tactile). There are also composite functions that are constructed using the low-level primitives. Examples of these functions are a command for the hand to grasp an object while maintaining specified tendon tensions and guarded move commands. On the second layer resides the DIAL control system. DIAL is a diagrammatic language that allows parallel processes to be represented in a compact graphical "time line." It was originally developed for the animation of graphical displays, but it has been transported by us to the robotics domain so we may exploit its ability to express parallel operations. It provides a convenient way to implement task-level scripts, which can then be bound to particular sensors, actuators and methods for accomplishing generic grasping or manipulation tasks. DIAL has proved to be an excellent tool for encoding parallel tasks and, because it is interpreted, it forms the basis of a powerful robotics development environment. Various stereotyped actions 


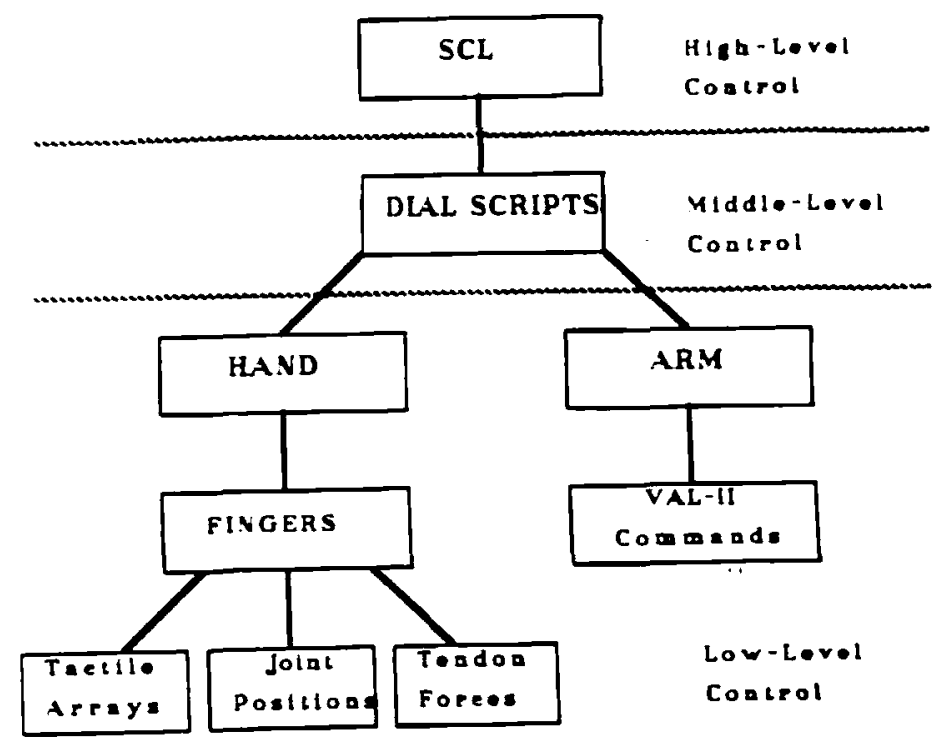

Figure 3: Control software overview

are programmed into DIAL scripts, such as edge followers and other actions that must be repeated within a task. We have added a third level above DIAL that allows us to reason with sensor data returned by DLAL scripts and to switch or repeat scripts based on sensory feed back. It also supports many high-level control abstractions, communications between processes, and asynchronous error handling. We have called this the Script Control Language (SCL). SCL is a block structured, interpreted language that has made it relatively easy to program the exploratory procedures described below. We have also used this system to demonstrate such tasks as pouring a liquid from a pitcher, picking up objects and moving them to new locations, and screwing in a light bulb. Although there are several layers within the system, the robot programmer need only work with DIAL scripts and SCL programs that invoke those scripts. Once the low-level primitive functions have been defined for a system, it is possible to program the robot at the highest levels. Figure 3 shows a block diagram of the control software for the system.

\subsection{The exploratory procedures}

The EP's that we have begun to investigate are those that yield estimates of global shape while being able to detect local surface features. Most notable are procedures implementing the enclosing and the contour following EP cited in Table 1. We are taking a hierarchical, coarse-to-fine approach to gathering shape information. Our approach is to find gross object shape initially and then use a hypothesis and test method to generate more detailed information about an object as discussed in Allen [All88]. This motivates the idea of using an initial global estimate of shape which can then be further refined by more specific and localized sensing. 
The problem of generating a good initial hypothesis is central to robust object recognition. If we can generate a good initial shape estimate, then we will be much more successful as we try to discover further object structure. The requirements for an initial shape estimator are that it be efficient, stable in the process of noise and uncertainty, and able to use sparse, partial data. We have implemented such a shape recovery method which we call exploration by enclosure. This method was initially discussed in [AR89] and it is reviewed briefly here, since it serves as a precursor to the other two EP's we have implemented.

\subsection{Exploration by enclosure}

Exploration by enclosure is an attempt to understand an object's gross contour and volume by effectively molding the hand to the object. This procedure was discussed by Gibson and Klatzky and Lederman. We have chosen to model objects as superquadrics [BS87] [Pen86] [Bar81]. Superquadrics form a rich set of shape primitives that allow a wide degree of freedom in modeling objects. Our work with shape recovery using superquadrics has been described fully in [AR89].

The PUMA arm moves the hand to a position in which it will close around the object. The fingers are spread wide during approach. Then the fingers are closed by position commands until the observed force (estimated by the difference between the flexor and extensor tendon tensions) exceeds a given threshold, which indicates that the finger is in contact with the object. The joint angle positions are read, and kinematic models of the hand and the PUMA arm are used to convert them to XYZ positions in world coordinates. Then the fingers are opened wide again, and a second containing grasp is executed, with the fingers taking different approach paths. The fingers are spread once again, and the PUMA arm moves the hand to the next position.

We tested this procedure against a database of 6 objects. The database included objects that could be modeled as undeformed superquadrics (block, large cylinder, small cylinder) and deformed (tapered) superquadrics (light bulb, funnel, triangular wedge). The recovered shapes are shown in Figure 4 with the sample data points overlaid on them.

The results of these experiments are quite good, especially considering the sparse nature of the data and the errors in the derived contact points. These errors are a function of the accuracy and calibration of the robotic arm, the hand joint position sensors, and the kinematic model of the hand itself. In spite of this sensor error, the recovered shapes are an accurate representation of the actual object's shape. The data points are overlaid on the recovered shapes to show the closeness of fit and the sparseness of the data. Each object's shape was recovered with extremely sparse amounts of data; typically 30-100 points, depending on the object. It is important to note that this is about two orders of magnitude less than typical range data images which try to recover shape with denser data, that, unlike touch sensing, is limited to a viewpoint that only exposes half the object's surfaces to the sensor. 

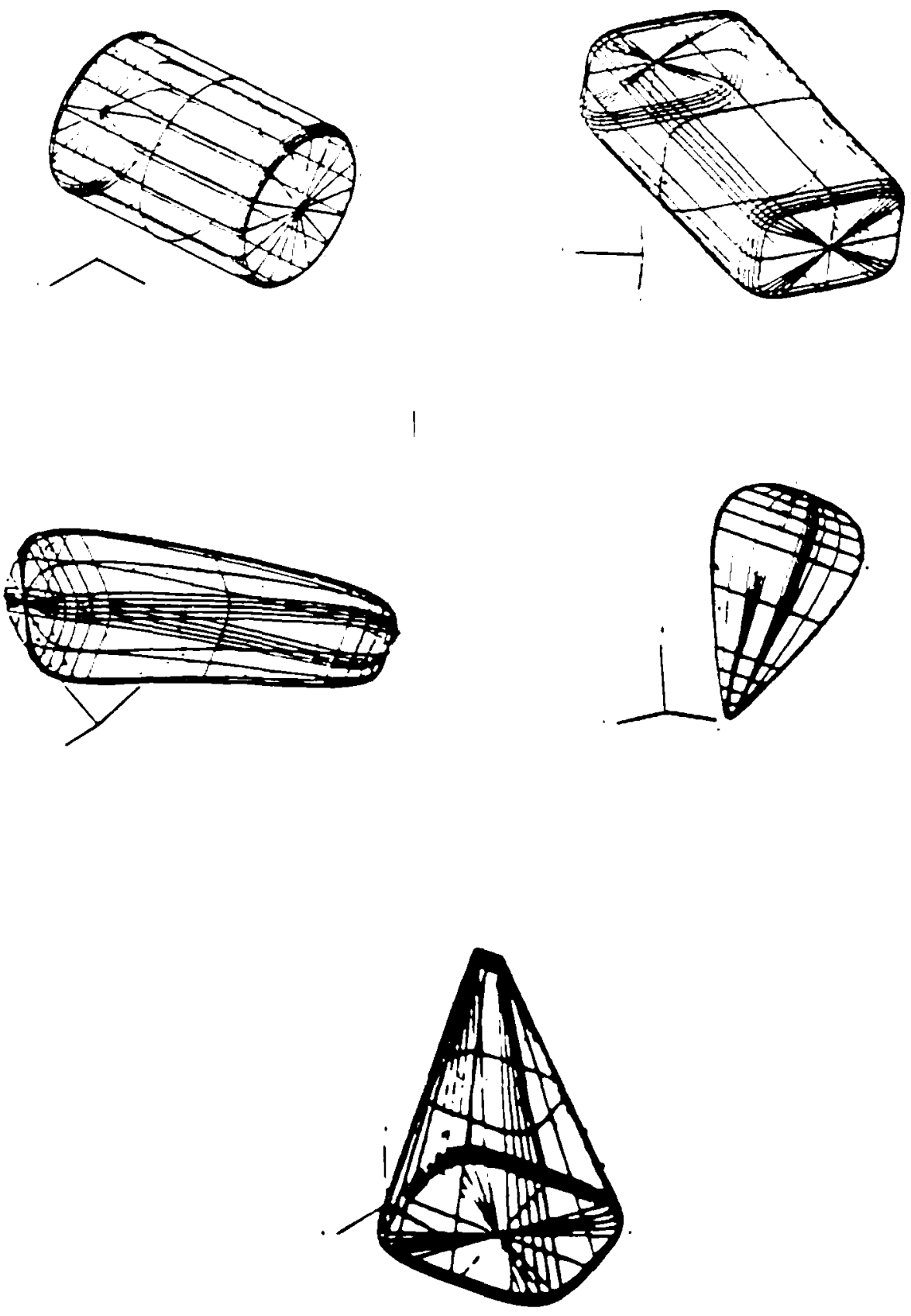

Figure 4: Recovered shapes of cylinder, block, wedge, light bulb, and funnel 


\subsection{Planar Surface Explorer}

Once a superquadric has been fit to the initial grasp data, we have a strong hypothesis about an object's shape. Of particular importance are the shape parameters. The shape of an object can be inferred from these parameters and used to direct further exploration. For example, if the shape parameters appear to be rectangular, then the planar explorer can trace out the plane and perform a least square fit of the trace data to test the surface's planarity. If the shape parameters appear more cylindrical, the planar faces of the cylinder can be explored with this primitive, and the cylinder's contour can be explored and verified with the contour follower EP (described below). A major benefit of using the superquadric analytic shape description is that it supplies orientation and axis data that are necessary for further active probes of the environment with the hand. Instead of a blind search, we can use the recovered orientation parameters to guide the further exploration of the object. Discovering a planar surface can be a very useful constraint in recognition, particularly if two opposing planar faces are grasped. By discovering multiple planar faces on an object, the recovery methods of Grimson and LozanoPerez [GLP83] and Ellis et al. [ERH86] cau be invoked, which provide strong constraints on recognition of an object.

The problem of using a tactile device to trace a surface on an object is a complicated one. Previous work by Allen [All88] using a one-fingered tactile sensor mounted on a PUMA traced along a curved surface by calculating a weighted vector of constraint directions that tried to follow the surface curvature while preserving smoothness of the trace and a constraint having to do with creating regions bounded by traces that were equivalent in size. Hor [Hor87] traced contours of planar objects using a planar four-fingered "chopstick"-like manipulator. Strain gauge sensors on the fingers of this device would calculate surface normals and move tangentially along a surface, recording the contour. Stansfield [Sta87] used a planar LORD tactile sensor mounted on a PUMA to trace edges and other features on objects.

We experimented with a single-finger scenario in which a finger maintains contact with a surface to find the surface normal. Although our work used a single finger, there is nothing to preclude the use of multiple fingers moving simultaneously over a surface. If two planar surfaces are found by this technique, then the controller could hypothesize that an edge had been detected and use another procedure to test this hypothesis. In our case, we assumed that a rough estimate of the orientation of a surface was given. Then the index finger moved until it contacted that surface, and the contact point in XYZ world coordinates was stored. After the initial contact was made, the arm was repeatedly moved in a direction hypothesized to be parallel to the face of the plane, and each tactile contact was duly noted. It is important that the points collected are not collinear, so the procedure that moves the arm makes certain that all points are not on the same line. If the edge of the object is found, then the finger is lifted off the surface and moved back to a known location. There are a number of ways in which the system can autonomously detect a discontinuity in the surface normal. Either the tactile sensors fail to detect contact even though the finger is flexed; or there is a dramatic change in the tendon force differentials between a valid contact point and one nearby; or when the poin $t$ is processed by the least squares fit program, it estimates that the point is not in the same plane. The acquisition of data points in this method is compatible with the three-point seed method of Henderson and Bhanu for forming planar surfaces from range data [HB82]. 


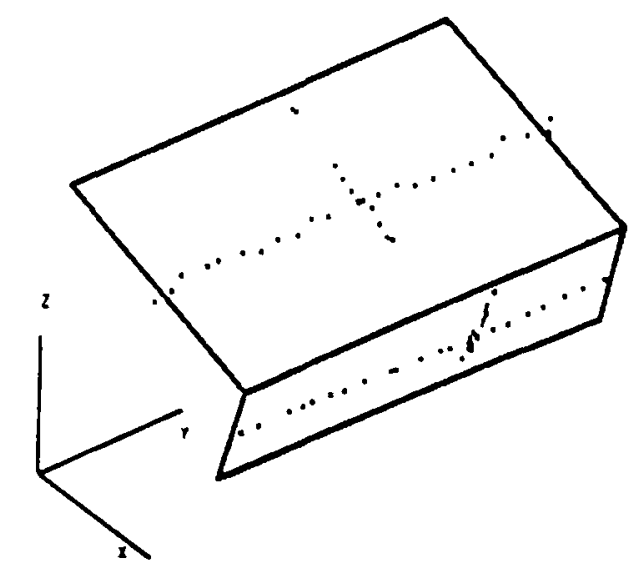

Figure 5: Planar Surface Explorer tactile contacts on 2 planar surfaces of a rectangular block.

Figure 5 shows a pattern of traces on 2 adjacent planar faces of a rectangular block using this EP. In our experiment, the search strategy employed was to move the finger along two lines that are perpendicular to each other. This procedure was repeated for two faces of a rectangular block. After the data were collected, the normals to the surfaces were computed by a least-square fit. The computed angle between the recovered planes is $96 \mathrm{deg}$ (the actual angle is unknown but assumed to be $90 \mathrm{deg}$ ). In the case of a simple rectangular block, it is easy to see how the edge linking to two explored faces can be hypothesized by intersecting the two planes.

\subsection{Surface Contour Following}

The third EP we have implemented is surface contour following with a two-fingered grasp. This EP allows us to determine an object's contour which has been shown to be a strong shape cue from previous vision research [PCM87]. The contours we are able to extract from touch are inherently three-dimensional. This simplifies recovery of shape since the 2-D image projection used in most contour work entails a loss of information. Since we can recover the three-dimensional contours, we are able to hypothesize a number of different shapes including generalized cylinders and solids of revolution, using the three-dimensional contour alone.

Our method is now described. First, the PUMA is moved to a location near one end of the explored object, and the thumb and index finger are opened enough to allow them to encompass the object without making contact with it. Then the thumb and index finger are slowly moved toward the object until the sensors detect contact between the fingertips and the object. After detecting contact, the positions of the two contact locations are noted, and the 
fingers are backed off the object so that they are no longer in contact. The arm and hand are moved a small amount along the axis of the explored object, and the process is repeated. This exploratory procedure ends when one of the fingers moves toward the object and fails to make contact. (The location of the object and its axis are not currently determined autonomously, but with human aid.)

The detection of contact and conversion to Cartesian coordinates is a process that requires several steps. To detect the tactile contact, two types of sensing are used: tactile, if the contact is in a region of the fingertip covered by a tactile sensor; and joint position, which signals contact when the actual and desired positions diverge while no contact is detected by the tactile sensors. To find the center of the contact, the first moments of the array are taken. Then a transformation is performed from the fingertip coordinate frame to the hand coordinate frame, and finally, from the hand coordinate frame to world coordinates. Currently, after detecting contact that does not involve the tactile sensor, the exploratory procedure continues looking for valid contact points along the original search axis.

We have performed a series of experiments to recover the shape of a number of different solids of revolution including a wine bottle, a beer bottle, a coke bottle and an Orangina soft drink bottle (a flask like object). The procedure begins by exploring the object along an exploration axis that is assumed to be perpendicular to the support table. The points generated from these contour traces are then linked into a set of linear contour segments. Circular cross section curves are then fit perpendicular to the exploration axis and including trace points from each of the contours. The recovered shapes are shown in Figure 6 . The shapes are clearly distinguishable from this sparse data. An additional and important discriminating characteristic is actual 3-D size and volume which are calculable from these representations.

\section{Summary}

We have presented the results of experiments dealing with active sensing with a dexterous robotic hand. These results have generally confirmed our belief that with a rich control system and a variety of different sensors, it would be possible to construct a system for robotic haptic perception.

In the future, we hope to add several new features. First, we will link the exploratory procedures together and integrate them into the high-level control so the entire process of shape recovery can become more autonomous. The addition of a vision system is necessary for this level of autonomy. To perform the contour following procedure, for example, vision will be used to determine an object's exploration axis.

\section{Acknowledgements}

This work was supported in part by DARPA contract N00039-84-C-0165, NSF grants DMC-86-05065, DCI-86-08845, CCR-86-12709, IRI-86-57151, North American Philips Laboratories, and the AT\&T Foundation. Thanks to Ken Roberts for helping acquire the superquadric recovery data and helping to set up and calibrate the hand, and Ajit Singh for his artistic skill. 

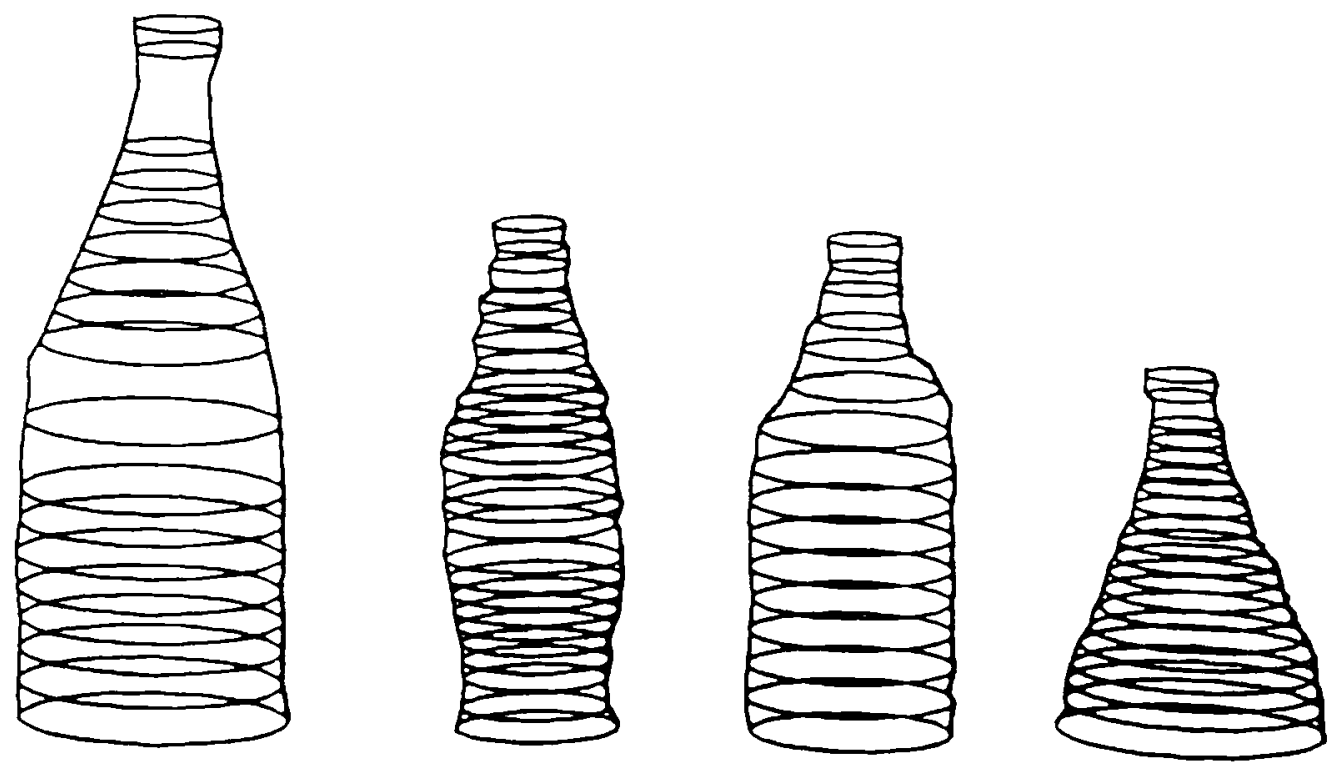

Figure 6: Recovered solids of revolution from surface contour explorer (left to right): wine bottle, coke bottle, beer bottle, Orangina bottle 


\section{References}

[All88] Peter Allen. Integrating vision and touch for object recognition tasks. International Journal of Robotics Research, 7(6):15-32, 1988.

[AMR89] Peter Allen, Paul Michelman, and Kenneth S. Roberts. An integrated system for dextrous manipulation. In 1989 IEEE International Conference on Robotics \& Automation, volume 1, pages 612-617, 1989.

[AR89] Peter Allen and Kenneth S. Roberts. Haptic object recognition using a multi-fingered dextrous hand. In 1989 IEEE International Conference on Robotics \& Automation, volume 1, pages 342-347, 1989.

[Bar81] Alan Barr. Superquadrics and angle preserving transformations. IEEE Computer Graphics and Applications, (1):11-23, 1981.

[BS87] Ruzena Bajcsy and Franc Solina. Three dimensional object representation revisited. In Proceedings International Conference on Computer Vision, June, 1987.

[BSD89] A. Bicchi, J.K. Salisbury, and P. Dario. Augmentation of grasp robustness using intrinsic tactile sensing. In 1989 IEEE International Conference on Robotics \& Automation, pages 302-307, 1989.

[Co.86] Interlink Co. The force sensing resistor: $A$ new tool in sensor technology. $535 \mathrm{E}$. Montecito, Santa Barbara, CA, 1986.

[ERH86] R. Ellis, Edward Riseman, and A.R. Hanson. Tactile recognition by probing: Identifying a polygon on a plane. In Proceedings of $A A A 1-86$, pages $632-637,1986$.

[GHM89] Roderic A. Grupen, Thomas C. Henderson, and Ian D. McCammon. A survey of general-purpose manipulation. International Journal of Robotics Research, 8(1):3862, February 1989.

[Gib66] J.J. Gibson. The Senses Considered as Perceptual Systems. Houghton Mifflin, 1966.

[GLP83] W.E.L. Grimson and Tomas Lozano-Perez. Model based recognition and localization from sparse three dimensional sensory data. Technical Report 738, M.I.T. A.I. Laboratory, 1983.

[Har82] Leon D. Harmon. Automated tacile sensing. International Journal of Robotics Research, 1(2):3-32, 1982.

[HB82] T.C. Henderson and Bir Bhanu. Three point seed method for the extraction of planar faces from range data. In Proc. of IEEE Workshop on Industrial Applications of Machine Vision, pages 181-186, 1982.

[HC89] R.D. Howe and M. Cutkosky. Sensing skin acceleration for slip and texture perception. In 1989 IEEE International Conference on Robotics \& Automation, pages 145-150, 1989.

[Hil82] W. Daniel Hillis. A high-resolution imaging touch sensor. International Journal of Robotics Research, 1(2):33-44, 1982. 
[Hor87] Maw-Kae Hor. Control and task planning for a four finger dextrous manipulator. PhD thesis, Courant Institute, New York University, October 1987.

[Igg82] A. Iggo. Cutaneous sensory mechanisms. In H.B. Barlow and J.D. Mollon, editors, The senses. Cambridge University Press, 1982.

[JIK+86] S.C. Jacobsen, E.K. Iversen, D.F.Knutti, R.T. Johnson, and K.B. Biggers. Design of the Utah/MIT dextrous hand. In 1986 IEEE International Conference on Robotics $\mathcal{E}$ Automation, pages 1520-1532, 1986.

[KL88] R.L. Klatzky and S. Lederman. Intelligent exploration by the human hand. In IEEE Workshop on Dexterous Robot Hands, pages 2-12, Philadelphia, PA, April 24, 1988.

[PCM87] J. Ponce, D. Chelberg, and W. Mann. Invariant properties of the projections of straight homogeneous cylinders. In Proc. First International Conference on Computer Vision, London, 1987.

[Pen86] Alex P. Pentland. Recognition by parts. Technical Report 406, SRI International, 1986.

[Sal82] J.K. Salisbury. Kinematic and Fonce Analysis of Articulated Hands. PhD thesis, Stanford University, July 1982.

[Spe88] Thomas Speeter. Flexible piezo-resistive touch sensing array. In SPIE Conference on Optics, Illumination and Image Sensing for Machine Vision III, Cambridge, November 1988.

[Sta86] S.A. Stansfield. Primitives, features, and exploratory procedures: bulding a robot tactile perception system. In 1986 IEEE International Conference on Robotics $\mathcal{O}$ Automation, pages 1274-1279, 1986.

[Sta87] S.A. Stansfield. Visually-guided haptic object recognition. PhD thesis, Department of Computer and Information Science, University of Pennsylvania, October 1987.

[TBK87] Rajko Tomovic, George A. Bekey, and Walter J. Karplus. A strategy for grasp synthesis with multifingered robot hands. In 1987 IEEE International Conference on Robotics \& Automation, pages 83-89, 1987.

[Tis88] B. Tise. A compact high resolution piezo-resistive digital tactile sensor. In 1988 IEEE International Conference on Robotics \& Automation, pages 760-764, 1988.

[UPB88] Nathan Ulrich, Richard Paul, and Ruzena Bajcsy. A medium-complexity compliant end effector. In 1988 IEEE International Conference on Robotics $\&$ Automation. pages $434-436,1988$.

[vBB86] H. van Brussel and H. Belien. A high resolution tactile sensor for part recognition. In Proceedings of the 6th International Conference on Robot Vision and Sensory Controls, Paris, June, 1986. 